\title{
A Transdisciplinary Approach for the Design Optimization of Medical Simulations
}

\author{
Agnese BRUNZINI ${ }^{\mathrm{a}, 1}$, Margherita PERUZZINI ${ }^{\mathrm{b}}$, Michele GERMANI ${ }^{\mathrm{a}}$, Daniele \\ MESSI $^{\mathrm{c}, \mathrm{d}}$, Pamela BARBADORO \\ ${ }^{a}$ Department of Industrial Engineering and Mathematical Sciences, Università \\ Politecnica delle Marche, Via Brecce Bianche 12, \\ 60131 Ancona, Italy \\ b Department of Engineering "Enzo Ferrari”, University of Modena and Reggio \\ Emilia, via Vivarelli 10, 41125 Modena, Italy \\ ${ }^{\mathrm{c}}$ Università Politecnica delle Marche, Via Tronto, 10/a, 60126 Ancona, Italy \\ d Azienda Ospedaliero Universitaria Ospedali Riuniti, 60126 Ancona, Italy \\ ${ }^{\mathrm{e}}$ Department of Biomedical Sciences and Public Health, Section of Hygiene, \\ Preventive Medicine and Public Health, Università Politecnica delle Marche, Via \\ Tronto, 10/a, 60126 Ancona, Italy
}

\begin{abstract}
Simulation in healthcare is rapidly replacing more traditional educational methods, becoming a fundamental step in the medical training path. Medical simulations have a remarkable impact not only on learners' competencies and skills but also on their attitudes, behaviors, and emotions such as anxiety, stress, mental effort, and frustration. All these aspects are transferred to the real practice and reflected on patients' safety and outcomes.

The design of medical simulations passes through a careful analysis of learning objectives, technology to be used, instructor's and learners' roles, performance assessment, and so on. However, an overall methodology for the simulation assessment and consequent optimization is still lacking.

The present work proposes a transdisciplinary framework for the analysis of simulation effectiveness in terms of learners' performance, ergonomics conditions, and emotional states. It involves collaboration among different professional figures such as engineers, clinicians, specialized trainers, and human factors specialists. The aim is to define specific guidelines for the simulation optimization, to obtain enhanced learners' performance, improved ergonomics, and consequently positively affect the patient treatment, leading to cost savings for the healthcare system. The proposed framework has been tested on a low-fidelity simulation for the training of rachicentesis and has allowed the definition of general rules for its enhancement.
\end{abstract}

Keywords. Transdisciplinary design, Design optimization, Simulation-based training, Human Factors, User experience.

${ }^{1}$ Corresponding Author, Mail: a.brunzini@staff.univpm.it. 


\section{Introduction}

For some decades, simulation has been used for educational purposes in several fields. It is defined as an imitation of a real system, able to mimic the real situation both in practical and behavioral contents. Indeed, its main advantage is the opportunity to involve the participants in a very immersive experience, avoiding the risks that would arise in real situations. For this reason, simulation is considered an excellent tool to reduce errors in high-risk industries such as aviation, defense, the nuclear energy field, and even healthcare.

The healthcare industry has begun to use simulation because it allows repeatedly practicing on simulated complications without putting the patient at risk, and thus, as a consequence, reducing the chance of bad outcomes in the real practice [1]. Moreover, beyond the learning of practical procedure and technical skills, simulations also offer the healthcare staff the opportunity to learn several non-technical skills such as clinical decision-making, situation awareness, team working, communication skills. For this reason, simulation-based training is considered an optimal solution for preparing and assessing human responses to real-life problems: it provides the opportunity to experience realistic training in terms of both clinical practice and stress management.

The stress role in the simulation context is crucially important together with the cognitive load. Indeed, during simulations, the participant is simultaneously exposed to the realism of the event, and to the demand to execute the correct intervention. Concerning the stress, while its feeling should be similar to that one felt in real practice, in the meantime, it should be maintained within certain limits, avoiding acute stress, which can compromise the performance, and post-traumatic stress disorders.

Regarding the mental effort, it should be balanced to avoid, on one side, low levels of attention, and, on the other side, cognitive overload which may compromise the learning path and the acquisition of new skills. In this context, the importance of a transdisciplinary approach to analyze the impact of medical simulations on trainees' stress, cognitive load, perceptions, workload, and performance is undeniable. Indeed, only with a comprehensive and transdisciplinary methodology, it would be possible to study the effectiveness of the simulation from the students' perspective.

Several definitions of transdisciplinarity exist, but all agree on the necessity to go beyond the scientific disciplines and interact with non-scientific expertise [2]. Ergonomics, which is indispensable in this context, is a classical transdisciplinary discipline because it blends technical and social aspects. Indeed, it concerns the design of systems, machines, or interfaces, based on the users' needs and requirements [3].

Several studies in the scientific literature analyzed the user experience, physical, and cognitive ergonomics with transdisciplinary approaches in the simulation context, but they are mainly applied to the aviation [4], and automotive [5] domains. Therefore, it emerges the need to apply such kind of transdisciplinary analysis also to the medical simulation training [6]. It would be extremely useful for the optimization and re-design of simulators and simulations, from a user-centered design perspective, and thus for the improvement of learners' performance, avoiding cognitive overloads and excessive stress. Only effective medical training can lead to improved clinical performance, patient safety, and, consequently, quality of care.

The paper focuses on defining a transdisciplinary framework for the analysis of simulation effectiveness in healthcare applications. It aims at measuring the quality of training in terms of performance as well as user experience (UX) considering the users' 
cognitive and emotional states of the trainees and defining specific guidelines to optimize the simulation-based training process.

\section{Research Background}

Nowadays, a standard definition and classification of simulations still do not exist. It can be classified into human (i.e. a role-play among students) or non-human simulation (e.g. using a manikin or computer), or according to the used educational tool [7]: standardized patients (with actors), screen-based computer (with interactive software), skill trainers (with partial manikins), high-fidelity manikins (with anatomical full-sized, computercontrolled simulators). It may be also classified according to fidelity [8], which is a multidimensional construct, related to the perception of how real or lifelike a simulator/simulation is for the user.

Different authors referred to various levels of fidelity. All agree in distinguishing between "engineering/physical fidelity" and "psychological fidelity" referring to the realistic look of the simulators in the first case, and to the demand of specific behaviors to complete the task in the second case [9]. Curtis et al. [10] considered also the "functional fidelity" involving actions, responses, and instrument accuracy. VincentLambert et al. [11] added three other dimensions: social, the culture of the group, and degree of openness or trust.

Psychological fidelity is considered very critical and more important for learning and transfer than the other dimensions [9]. Accurately reproducing stressful conditions is one of the most fundamental and challenging aspects of simulation design. During the design of simulations and simulators, great attention must be placed on the realism and feeling of immersion; for this reason, a close collaboration between physicians and engineers who design the simulator is always needed. Also, the inclusion of pedagogical and psychological expertise into the design and development of educational devices is essential [12].

To have successful simulation-based training, above the simulated scenario, also briefing and debriefing must be carefully considered during the design. The briefing takes place before the simulation; in this period the teacher explains the objectives of the simulation and how it will be conducted. The debriefing occurs after the simulation and is an active retrospective assessment in which students appraise their technical and cognitive performance, highlighting the importance of human factors [13]. For its nature, it is considered the most important phase of the simulation. Debriefing is developed as a therapeutic practice for people experiencing traumatic and stressful events. Discussing shared experiences in terms of practical performance, potential wrong approaches, and related feelings is a strategy to minimize the chance of post-traumatic stress disorders [14].

Therefore, simulation-based training is characterized by a pedagogical framework that allows the students to experiment with the same workflow and workload that they would experience in real clinical cases [8]. The design of medical simulations passes through a careful analysis of learning objectives, technology to be used, instructor role, performance assessment, and so on.

However, a series of issues need further research: among these, the development and use of transdisciplinary assessment tools for the evaluation of technical and behavioral competencies $[15,16,17]$ arise. 
Human Factors (HF) and Ergonomics have been introduced in engineering to add the physical, psychological, social, and cultural needs of human beings, to the mechanical, electrical, manufacturing requirements considered during the product/system design [18]. Moreover, HF is fundamental also in the evaluation of the human-machine interaction and the evaluation of the UX, helping in the improvement of human performance, the optimization of physical and mental workload, comfort, and perceived effort, and the reduction of the risks associated with user errors.

However, even if education in healthcare focuses on high-stakes environments and the acquisition of complex manual and cognitive skills, human factors are not well integrated and adopted into medical training [19]. Indeed, the number of successful implementation and development of medical simulations is relatively small compared with the manufacturing industry.

In this context, the adoption of a human-centered, transdisciplinary approach is compulsory for the creation of successful training paths and simulators, and their optimization and re-design.

\section{Research Approach}

\subsection{Transdisciplinary Framework}

To assess medical simulation effectiveness, several transdisciplinary aspects have to be analyzed before, during, and after the simulation itself (Figure 1). Based on this accurate and extensive assessment, it is then possible to outline the recommendations to re-design and optimize the simulation.

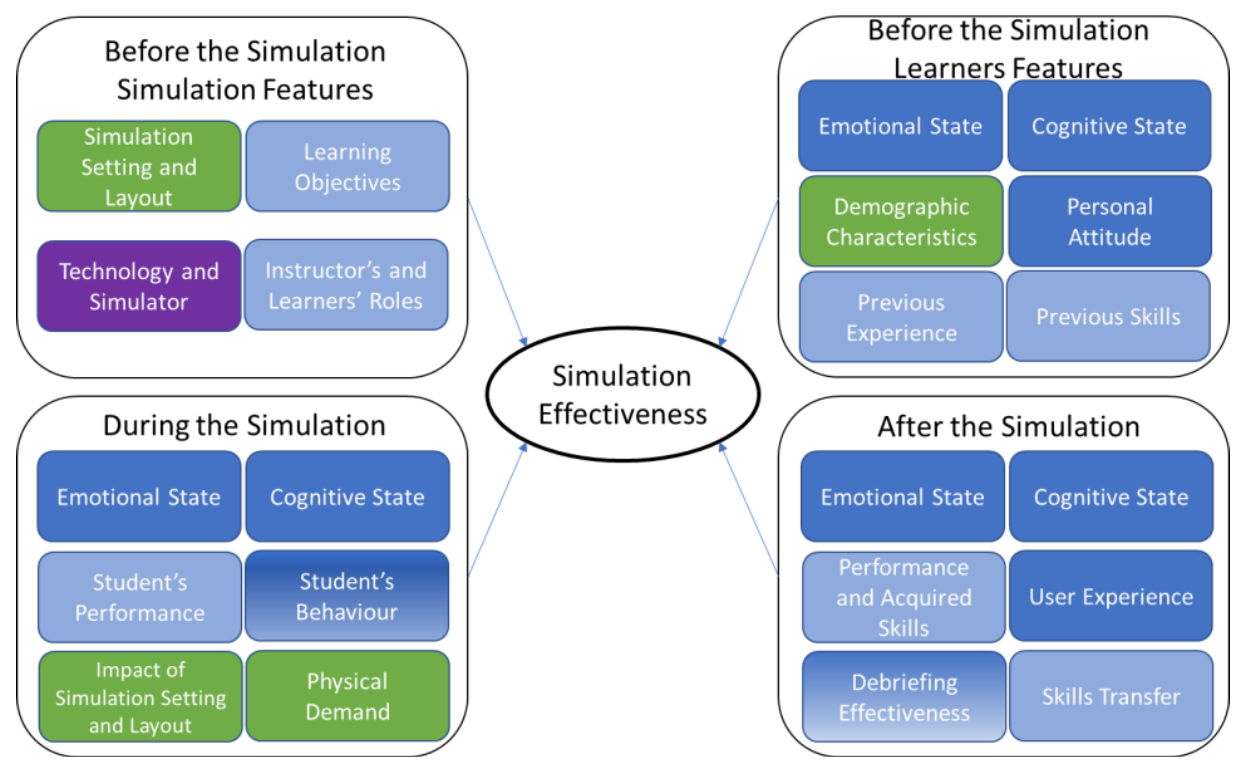

Figure 1: Transdisciplinary Framework for the assessment of medical simulation effectiveness. Included expertise: technical / engineering (purple), ergonomics (green), pedagogical (light blue), psychological (blue), or a blend of both (blue/light blue) 
Before the accomplishment of the simulation, the engineering, pedagogical, and social aspects must be considered. The first ones relate for example to the technical aspects of the used simulator, the second ones to the predefined learning goals, the roles of learners and instructor during the simulation, and the third ones to the demographic, and personal characteristics of learners (such as previous experiences, skills, attitude,...).

The cognitive conditions and emotional states (such as stress, anxiety, frustration, effort,..) of learners must be considered before, during, and after the simulation, to understand its effect on such conditions. According to ISO 10075-3, 2004 [20], the mental workload can be measured through performance assessment methods, selfassessment methods, and physiological measurements methods. Among the three different assessment techniques, the self-assessment method is the most applied in the healthcare simulation field [21]. However, subjective user ratings can be misleading metrics of simulation effectiveness [10]. Examples of the application of physiological measurements exist in the literature [22], but they are not widespread. For this reason, it is evident the need of including a multi-dimensional assessment.

Also, for the detection of psychological stress four main criteria are used [20]: psychological, physiological, behavioral, and biochemical. The most common analyses typically include the subjective assessment based on self-report and physiological assessment based on heart rate, and skin conductivity monitoring.

Moreover, even the ergonomics of the simulation room and instruments' layout must be considered both in relation to the cognitive and physical demands for learners.

After the end of the simulation, it is important to acquire information about the user experience, the acquired skills and performance, and the effectiveness of the debriefing phase on the learners' stress and cognitive conditions.

\subsection{Data Analysis}

Several assessment measures can be exploited to analyze the different research areas included in the transdisciplinary framework:

- Technical area (which includes the technical aspects of the used simulator and technology): in a user-centered perspective, the usability and user experience related to the use of the simulator and/or advanced technologies (e.g. extended reality devices) should be assessed through ad-hoc questionnaires and usability tests;

- Ergonomics area (which comprehends simulation setting and layout, physical demand, and demographic characteristics): the physical ergonomics should be analyzed by using wearable sensors (e.g. for the acquisitions of the EMG signal, motions, etc.) or virtually through different digital human models. Also, the learners' demographic characteristics (i.e. age, weight, height, etc.) should be collected and studied through statistical regression models to understand their effect on the simulation execution.

- Pedagogical area (that includes learning objectives, instructor's and learners' roles, previous experience and skills, performance, acquired skills, and skill transfer): the performance should be evaluated during the simulation by using specific checklists and recording times, errors, attempts, etc. The acquisition of skills should be assessed both with a comparison between the pre- and postsimulation and then in the clinical field with real medical cases. 
- Psychological area (which comprehends emotional and cognitive states and personal attitude): feelings such as frustration, anxiety, effort, etc. could be assessed through self-assessment, comparing the learners' perception before and after the simulation, and then through statistical analysis. The cognitive load and the stress could be monitored in real-time using smart wearable devices for the acquisition of physiological parameters. In this way, it is possible to discern between perceived and physiological cognitive/emotional states and understand the variables that most affect the simulation effectiveness.

Therefore, thanks to the application of this multi-dimensional analysis and the use of different assessment methods, it is possible to study:

- Perceived stress, anxiety, frustration, mental effort through the self-assessment questionnaires: variation between pre- and post- simulation.

- Objective stress and cognitive load based on the continuous physiological monitoring (e.g. heart rate, heart rate variability, breathing rate, electrodermal activity, ...) before, during, and after the simulation (i.e. comparison between stressful and restful tasks or simulation phases).

- Differences in learners' opinions about the usefulness of simulation-based training.

- Usability of the simulator and/or other technologies and user experience of the learners (in relation to the simulation layout too).

- Performance and skills evaluation for the single tasks or the overall simulation.

- Standard central trend measures for the description of the demographic characteristics, the performance, the biometric indices, as well as for the analysis of the responses to the self-assessment questionnaires.

- Statistical single and multiple linear regression analysis to discover the variables that affect students' performance, stress, cognitive load, during the simulation-based training.

Based on these relationships among variables, it is possible to define specific guidelines for the optimization and re-design of the simulations, to improve the simulation effectiveness and balance the levels of stress and cognitive load.

\section{Use Case}

\subsection{Analysis}

This study considered the low-fidelity simulation for the training of rachicentesis, which is a specific lumbar puncture for the collection of the liquor, for diagnostic purposes. The goal of the simulation is to learn how to perform the puncture and become able to let the liquor spilling out. Indeed, if the needle is non-inserted in the right place and with the right depth, the liquor would not come out.

The simulation duration varies from a few minutes to half an hour, according to the learner's skills and ability. All the variables related to the simulation setting, used technology, simulation tasks, learners' characteristics have been recorded. The room layout included a desk with the skill trainer for the lumbar puncture and, on its right, all the needed instrumentation for the procedure.

In this case study, the standard simulation workflow (i.e. briefing, simulation, and debriefing) was not followed. Indeed the simulation was preceded by the theory explanation, the teacher briefing, and demonstration, but it was not followed by the 
debriefing. This work focused on the assessment of learners' performance, cognitive and emotional conditions, following the workflow in Figure 2.

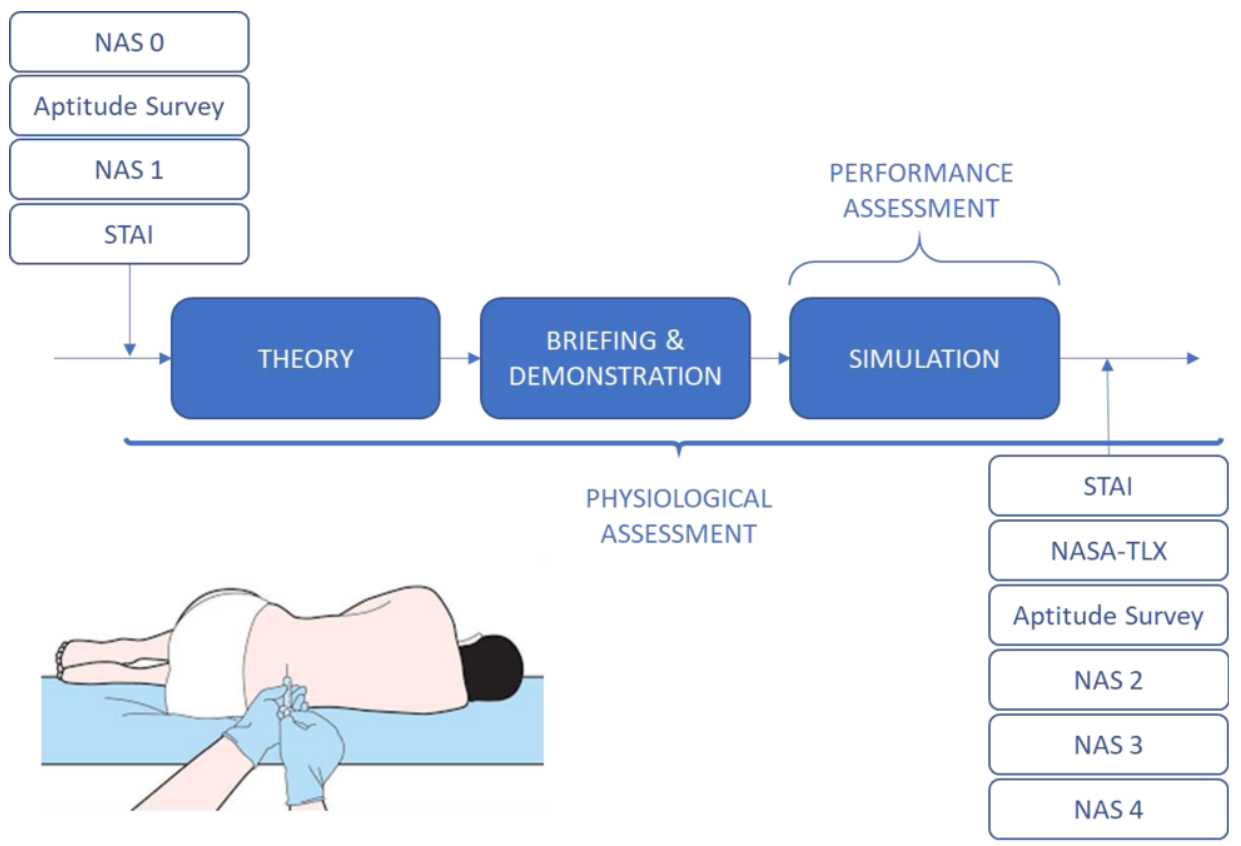

Figure 2: Simulation assessment workflow

The physiological parameters have been collected from the arrival in the classroom until the end of the simulation, through non-invasive wearable devices, to assess the objective levels of stress and cognitive load experienced by the learners, before, during, and after the training.

Several questionnaires were administered before and after the simulation to assess the subjective stress, cognitive load, anxiety, frustration, effort, and workload perceived by the learners, and to distinguish their variations due to the training.

The Numerical Analogue Scale (NAS) was administered at the arrival in the classroom, ten minutes later, and ten, twenty, and thirty minutes after the end of the simulation, to study the trend of the perceived stress.

The double-module State-Trait Anxiety Inventory (STAI) was administered to evaluate the anxious trait of learners in their lives (only before the simulation) and the perceived level of anxiety in those precise moments (before and after the training).

The NASA Task Load Index (NASA-TLX) was administered after the simulation to record the learners' opinions about the perceived mental, physical, temporal demands, effort, performance, and frustration.

The aptitude survey was administered before and after the training to understand the learners' aptitude and familiarity with simulations and advanced technologies.

Moreover, the performance was analyzed for each task of the entire procedure, in terms of committed errors, the number of attempts, consultations with the instructor, times, task correctly/incorrectly performed, or not performed. Also, a skill questionnaire was administered before and after the simulation, to verify the learning. 


\subsection{Results}

The self-assessment questionnaires and the physiological signals were singularly analyzed, as well as the performance. All these variables were then assessed through several models of multiple linear regression analysis. The statistical analysis highlighted that learners' performance is not influenced by perceived and physiological stress. Cognitive load and stress levels are well balanced during the simulation and they turn back to basal levels after the end of the training (suggesting that the debriefing is not indispensable in low-stressful simulations).

However, some issues emerged. Table 1 proposes some basic guidelines to solve the principal simulation's issues (related to learners' performance, cognitive and emotional conditions) highlighted by the multiple linear regression analysis.

Table 1. Possible solutions to simulation issues.

\begin{tabular}{|c|c|}
\hline & Solutions to issues \\
\hline \multicolumn{2}{|l|}{ Performance } \\
\hline $\begin{array}{l}\text { (Too) high cognitive load causes: } \\
\text { - Increment of simulation time } \\
\text { - Increment of errors }\end{array}$ & $\begin{array}{l}\text { Feedback about the tasks' sequence and execution } \\
\text { could be provided during the simulation }\end{array}$ \\
\hline $\begin{array}{l}\text { Success is not reached if the instructor practically } \\
\text { assists the learner }\end{array}$ & $\begin{array}{l}\text { The instructor should only assist the learner with } \\
\text { advice, without interrupting his/her simulation } \\
\text { execution }\end{array}$ \\
\hline Performance is worse in the afternoon & $\begin{array}{l}\text { Lesson contents could be divided into two sessions: } \\
\text { theory in the morning and simulation in the } \\
\text { afternoon (real cases could happen every time of the } \\
\text { day) }\end{array}$ \\
\hline \multicolumn{2}{|l|}{ Cognitive and Emotional States } \\
\hline $\begin{array}{l}\text { Anxiety before the simulation compromises } \\
\text { performance and learning }\end{array}$ & $\begin{array}{l}\text { The instructor should calm down the learners during } \\
\text { the briefing }\end{array}$ \\
\hline Stress is higher when more errors are committed & $\begin{array}{l}\text { If too many errors are committed, the instructor } \\
\text { should give theoretical help }\end{array}$ \\
\hline $\begin{array}{l}\text { For a long simulation duration: } \\
\text { - } \quad \text { Stress increases } \\
\text { - } \quad \text { Frustration increases } \\
\text { - } \\
\text { Effort increases }\end{array}$ & $\begin{array}{l}\text { The simulation duration could be reduced (and } \\
\text { consequently even stress, effort, and frustration) by } \\
\text { providing feedback that could help the learners in } \\
\text { the correct execution of the tasks }\end{array}$ \\
\hline The effort is higher for tall subjects & Physical ergonomics should be studied and adjusted \\
\hline
\end{tabular}

Therefore, from this transdisciplinary effectiveness analysis, the necessity of optimizing the simulation from pedagogical, psychological, ergonomic perspectives arises.

\section{Conclusions}

This work underlined the relevance of the application of transdisciplinary approaches for the design and optimization of simulation-based medical training. Indeed, in the 
simulation context, all the pedagogical, technical, ergonomic, and psychological dimensions assume a great weight. As it is important to achieve high performance through a valuable pedagogical path, it is also fundamental to assure a good stress balance: simulation should bring learners to a stress level similar to that one in the real practice, and, at the same time, it should not provoke excessive stress which may damages performance, consequently resulting in increased risk for the patient.

The same reasoning should be done for the cognitive load. Indeed, to guarantee better performance and the best skills memorization, the mental effort should not be too low, and, at the same time, cognitive overload must be avoided. Also, the physical domain must be considered: the learners should feel physically comfortable during the simulation.

Moreover, beyond the aspects treated in this work, even the technical features of the simulators and the level of fidelity should be taken into account, both from the psychological and engineering points of view.

\section{References}

[1] S. L. Dawson and J. A. Kaufman, The imperative for medical simulation, In: Proceedings of the IEEE, Vol. 86, pp. 479-483, 1998.

[2] M. Goudswaard, B. Hicks, and L. Newnes, An Appraisal and Classification of the Transdisciplinarity of Existing Design Tools, Advances in Transdisciplinary Engineering, Vol 12, pp. 50 - 59, 2020.

[3] F. Grandi, M. Peruzzini, C. E. Campanella, M. Pellicciari, Application of Innovative Tools to Design Ergonomic Control Dashboards, Advances in Transdisciplinary Engineering, Vol. 12, pp. 193-200, 2020.

[4] A.R. Hidalgo-Muñoz, D. Mouratille, N. Matton, M. Causse, Y. Rouillard, R. El-Yagoubi. Cardiovascular correlates of emotional state, cognitive workload and time-on-task effect during a realistic flight simulation. International Journal of Psychophysiology, Vol. 128, pp.62-69, 2018.

[5] F. Grandi, M. Peruzzini, R. Raffaeli, And M. Pellicciari, Transdisciplinary Assessment Matrix to Design Human-Machine Interaction, Advances in Transdisciplinary Engineering, Vol. 12, pp. 183-192. 2020.

[6] A. Brunzini, A. Papetti, E. Brandoni Serrani, M. Scafà, M. Germani, How to Improve Medical Simulation Training: A New Methodology Based on Ergonomic Evaluation, In: International Conference on Applied Human Factors and Ergonomics (AHFE 2019), AISC 96, pp. 145-155, 2020.

[7] S. Sahu and I. Lata, Simulation in resuscitation teaching and training: an evidence-based practice review, Journal of Emergencies, Trauma and Shock, Vol. 3, pp. 378-384, 2010.

[8] S.S. Elshama, How to apply Simulation-Based Learning in Medical Education? IberoAmerican Journal of Medicine, Vol. 02, pp. 79-86, 2020.

[9] G. Norman, K. Dore and L. Grierson, The minimal relationship between simulation fidelity and transfer of learning, Medical Education, Vol. 46, pp. 636-647, 2012.

[10] M.T. Curtis, D. Granados, and M. Feldman, Judicious Use of Simulation Technology in Continuing Medical Education, Journal of Continuing Education in the Health Professions, Vol. 32, pp. 255-260, 2012.

[11] C. Vincent-Lambert and F. Bogossian F, A guide for the assessment of clinical competence using simulation, U21 Health Sciences Group, 2017.

[12] A. Holzinger, M.D. Kickmeier-Rust, S. Wassertheurer, M. Hessinger, Learning performance with interactive simulations in medical education: Lessons learned from results of learning complex physiological models with the HAEMOdynamics SIMulator, Computers \& Education, Vol. 52, pp. 292$301,2009$.

[13] S.J. Mercer, Using full immersive simulation to prepare trauma teams to work in a major trauma centre, Trauma, Vol. 19, pp. 277-285, 2017.

[14] S. D. AL Sabei and K. Lasater, Simulation debriefing for clinical judgment development: A concept analysis, Nurse Education Today, Vol. 45, pp. 42-47, 2016.

[15] W.F. Bond, R.L. Lammers, L.L. Spillane, R. Smith-Coggins, R. Fernandez, M.A. Reznek, J.A. Vozenilek, J.A. Gordon, Society for Academic Emergency Medicine Simulation Task Force, The Use of Simulation in Emergency Medicine: A Research Agenda, Academic Emergency Medicine, Vol. 14, pp. 353-364, 2007. 
[16] T. Sawyer and M.M. Gray, Procedural training and assessment of competency utilizing simulation, Seminars in Perinatology, Vol. 40, pp. 438-446, 2016.

[17] S.L. Hanshaw and S.S. Dic, High fidelity simulation evaluation studies in nursing education: A review of the literature, Nurse Education in Practice, Vol. 46, 2020.

[18] ISO 9241-210, Ergonomics of human system interaction - Part 210: Human-centered design for interactive systems, 2010.

[19] F.J. Seagull, Human Factors Tools For Improving Simulation Activities In Continuing Medical Education, Journal Of Continuing Education In The Health Professions, Vol. 32, pp. 261-268, 2012.

[20] ISO 10075-3, Ergonomic principles related to mental workload // Part 3: Principles and requirements concerning methods for measuring and assessing mental workload, 2004.

[21] M. Scafà, E. Brandoni Serrani, A. Papetti, A. Brunzini, M. Germani, Assessment of Students' Cognitive Conditions in Medical Simulation Training: A Review Study, In: International Conference on Applied Human Factors and Ergonomics (AHFE 2019), Advances in Intelligent Systems and Computing, Vol. 958, pp. 224-233, 2020.

[22] A. Brunzini, A. Papetti, L. Formenti, A. Luciani, D. Messi, E. Adrario, P. Barbadoro, Cognitive Load and Stress Assessment of Medical High-Fidelity Simulations for Emergency Management, In: International Conference on Applied Human Factors and Ergonomics (AHFE 2020), pp. 343-350, 2021. 